OPEN ACCESS

Edited by:

Alexandre Gramfort, Université Paris-Saclay, France

Reviewed by:

Christian-G Bénar, Institut de Neurosciences des Systèmes (INSERM), France Erika June Christina Laing, UPMC Presbyterian, USA

*Correspondence:

Emilie Bourel-Ponchel emilie.boure/@u-picardie.fr

Specialty section: This article was submitted to Brain Imaging Methods, a section of the journal Frontiers in Neuroscience

Received: 20 May 2016 Accepted: 26 January 2017 Published: 10 February 2017

Citation: Bourel-Ponchel E, Mahmoudzadeh M, Berquin P and Wallois F (2017) Local and Distant Dysregulation of Synchronization Around Interictal Spikes in BECTS.

Front. Neurosci. 11:59. doi: 10.3389/fnins.2017.00059

\section{Local and Distant Dysregulation of Synchronization Around Interictal Spikes in BECTS}

\author{
Emilie Bourel-Ponchel ${ }^{1,2 *}$, Mahdi Mahmoudzadeh ${ }^{1,2}$, Patrick Berquin ${ }^{1,3}$ and \\ Fabrice Wallois ${ }^{1,2}$
}

${ }^{1}$ Institut National de la Santé et de la Recherche Médicale U 1105, GRAMFC, CURS, CHU Amiens Picardie - Site Sud, Salouël, Amiens, France, ${ }^{2}$ Fonctional Exploration of the Pediatric Nervous System, CHU Amiens Picardie - Site Sud, Salouël, Amiens, France, ${ }^{3}$ Neuropediatry Unit, CHU Amiens Picardie - Site Sud, Salouël, Amiens, France

Objective: High Density electroencephalography (HD EEG) is the reference non-invasive technique to investigate the dynamics of neuronal networks in Benign Epilepsy with Centro-Temporal Spikes (BECTS). Analysis of local dynamic changes surrounding Interictal Epileptic Spikes (IES) might improve our knowledge of the mechanisms that propel neurons to the hypersynchronization of IES in BECTS. Transient distant changes in the dynamics of neurons populations may also interact with neuronal networks involved in various functions that are impaired in BECTS patients.

Methods: HD EEG (64 electrodes) of eight well-characterized BECTS patients (8 males; mean age: 7.2 years, range: 5-9 years) were analyzed. Unilateral IES were selected in 6 patients. They were bilateral and independent in 2 other patients. This resulted in a total of 10 groups of IES. Time-frequency analysis was performed on HD EEG epochs around the peak of the IES ( $\pm 1000 \mathrm{~ms})$, including phase-locked and non-phase-locked activities to the IES. The time frequency analyses were calculated for the frequencies between 4 and $200 \mathrm{~Hz}$.

Results: Time-frequency analysis revealed two patterns of dysregulation of the synchronization between neuronal networks preceding and following hypersynchronization of interictal spikes ( $\pm 400 \mathrm{~ms})$ in the epileptogenic zone. Dysregulation consists of either desynchronization $(n=6)$ or oscillating synchronization $(n=4)(4-50 \mathrm{~Hz})$ surrounding the IES. The 2 patients with bilateral IES exhibited only local desynchronization whatever the IES considered. Distant desynchronization in low frequencies within the same window occurs simultaneously in bilateral frontal, temporal and occipital areas $(n=7)$.

Significance: Using time-frequency analysis of HD EEG data in a well-defined population of BECTS, we demonstrated repeated complex changes in the dynamics of neuronal networks not only during, but also, before and after the IES. In the epileptogenic zone, our results found more complex reorganization of the local network than initially thought. In line with previous results obtained at a microscopic or macroscopic level, these changes suggested the variability strategies of neuronal assemblies to raise IES. 
Distant changes from the epileptogenic zone in desynchronization observed in the same time window suggested interactions between larger embedded networks and opened new avenues about their possible role in the underlying mechanism leading to cognitive deficits.

Keywords: BECTS, time-frequency analysis, interictal epileptic spikes, desynchronization, hypersynchronization, pre-spike

\section{INTRODUCTION}

Benign Epilepsy with Centro-Temporal Spikes (BECTS) is the most common form of idiopathic childhood epilepsy with a prevalence of $15 \%$ in epileptic children aged $1-15$ years (Panayiotopoulos et al., 2008). BECTS, more common in boys, with a sex ratio 6/4 (Wirrell, 1998) is characterized by brief, hemifacial sensori-motor seizures that typically originate in the centro-temporal area (Beaussart, 1972; Loiseau and Beaussart, 1973) associated with interictal epileptic spikes (IES) localized in the unilateral and/or bilateral centro-temporal areas, which are typically activated by drowsiness and slow nonRapid eye movement (REM) sleep (Beaumanoir et al., 1974). The characteristics of the IES in BECTS constitute a clinical biomarker of this epilepsy. Well-defined IES in BECTS patients can be modeled by single tangential dipole sources oriented from central to frontal lobes and localized in the central regions (suprasylvian) (Ishitobi et al., 2005). Despite the infrequent seizures and the focality of IES in BECTS, cognitive, and/or behavioral disorders have been repeatedly reported in BECTS patients (Vannest et al., 2015). An implication of IES in cognitive deficits has been suggested, but the underlying neurophysiological mechanisms remain poorly understood.

The mechanisms that propel neurons to the hypersynchronization of the IES are multiple, involving synaptic and non-synaptic interactions (De Curtis and Avanzini, 2001) and changes in the immediate cellular configuration (Manoochehri et al., 2017) and hemodynamic environment (Jacobs et al., 2009b; Osharina et al., 2010). Based on intracerebral multi-unit recording in refractory epilepsy, Keller and collaborators suggested that the interictal epileptiform activity in patients with epilepsy is not a simple paroxysm of hypersynchronous excitatory activity, but rather represents interplay of multiple distinct neuronal types within complex neuronal networks (Keller et al., 2010). IES would result from complex interactions within different neuronal populations whose activities decrease or increase not only during the IES but also before and after the IES by a few $100 \mathrm{~ms}$ (Keller et al., 2010). At a macroscopic level, EEG and fMRI studies have

\footnotetext{
Abbreviations: BECTS, Benign Epilepsy with Centro-Temporal Spikes; ECoG, ElectroCorticoGraphy; EEG, ElectroEncephaloGraphy; fMRI, functional Magnetic Resonance Imagery; FOS, Fast Optical Signal; FPR, False positive rate; GTFR, Global Time-Frequency Representation; HD EEG, High Density EEG; HFOs, High-Frequency Oscillations; ITFR, Induced Time-Frequency Representation; IES, Interictal Epileptic Spikes; MUA, MultiUnit Activity; REM, Rapid Eye Movement; TCI, Tansient Cognitive Impairment; TFR, Time-Frequency Representation; sLORETA, Standardized low resolution brain electromagnetic tomography; SSLOFO, Standardized Shrinking LORETA-FOCUSS; WISC, Wechsler Intelligence Scale for Children.
}

demonstrated that IES are associated with complex network interaction not only inside the epileptogenic zone but also in distant areas including the frontal and temporo-occipital cortices (Cataldi et al., 2013; Fahoum et al., 2013; Adebimpe et al., 2015a,b, 2016) with extensive functional (Besseling et al., 2013; Tang et al., 2014; Adebimpe et al., 2015a,b, 2016) and structural changes (Garcia-Ramos et al., 2015) in cerebral activities.

BECTS patients are not accessible to intra-cerebral multiunit recording. To address the complexity of neurophysiological mechanisms around the IES in BECTS, scalp HD EEG in the time-frequency domain was analyzed. We expected that, like in refractory epilepsies, (Keller et al., 2010; Jacobs et al., 2011), IES are associated with complex changes in synchronization, which could precede IES. Moreover, because time analysis of scalp HD EEG allowed analyzing cortical activity more globally, at a macroscopic level, we thought that TFR could contribute to a better understanding of the interaction between the epileptogenic zone and distant areas.

The present study was designed to investigate the dynamics of large-scale neuronal networks by non-invasive analysis of changes in synchronization around the IES based on timefrequency analysis of High-Density electroencephalography (HD EEG) in a homogeneous male population of BECTS children.

\section{MATERIALS AND METHODS}

\section{Patients}

Eight male patients with typical BECTS (mean age: 7.2 years, range: 5-9 years) were included in the study. BECTS was diagnosed on the basis of a typical clinical history and the presence of characteristic IES on standard EEG, according to ILAE criteria (Berg et al., 2010).

Clinical diagnostic criteria of BECTS included children presenting sensori-motor seizures with inconsistent secondary generalization, with an age of onset between 4 and 10 years (Beaumanoir et al., 1974) and typical diphasic spikes either isolated or occurring in clusters, unilaterally or bilaterally, in the centro-temporal areas on a standard normal background EEG (Beaumanoir et al., 1974). Patients with an abnormal neonatal history, intellectual deficit (IQ $<70$ ), neurological abnormalities on physical examination, and/or any lesions in brain neuroimaging were not included in the study.

\section{Ethical Considerations}

The study was approved by the local ethic committee (CPP NordOuest, No. A00782-39). Written Informed consent to participate in the study was obtained from the parents and all patients before inclusion. 


\section{EEG Acquisition}

High-Density EEG were acquired with $\mathrm{Ag} / \mathrm{AgCl}$ electrodes $(n=64)$, disposed according to the $10 / 10$ international system (EasyCap ${ }^{\circledR}$ ). The EEG was recorded by eemagine EEG software (eemagine Medical, Imaging Solutions GmbH, Berlin, Germany) and sampled at $1024 \mathrm{~Hz}$ (ANT Inc., Enschede, The Netherlands), in DC mode. Only a notch filter $(50 \mathrm{~Hz})$ was applied. A mastoid reference was used for acquisition.

HD EEG recordings were performed during quiet arousal. The electrode impedances were kept below $5 \mathrm{k} \Omega$. The signals were rereferenced to an average reference for further analysis. Patients were monitored for movements during the acquisition, so that altered data could be later excluded.

\section{EEG Analysis}

\section{Interictal Epileptic Spike Selection and Artifact Rejection}

For IES selection, artifact rejection and all subsequent analyses, data were arithmetically re-referenced to average reference. Because, the spike complex BECTS can be explained by one tangential source (Pataraia et al., 2008; Kakisaka et al., 2011), we used scalp potentials. Indeed, tangential sources are likely located in fissures and sulci, thus deeper than radial sources. Surface Laplacians are more sensitive to radial than tangential dipoles. Sources in sulci will be therefore minimized and further reducing their contribution to a surface Laplacian. Moreover, the scalp surface Laplacian, which may be interpreted as a method which minimizes volume-conduction effects (important for connectivity analyses), does require accurate spline interpolation and may be sensitive to the choice of the spline parameters $(\mathrm{He}$, 1999).

Visual selection of IES containing similar spatial distribution, shape, and morphology is difficult and is associated with errors. To increase the accuracy of selection, IES were semiautomatically selected after reviewing the EEG with $B E S A^{\circledR}$ software.

First, typical BECTS spikes, characterized by diphasic or triphasic patterns distributed in the centro-temporal areas were selected manually (Beaumanoir et al., 1974). Second, IES sets containing similar spatial distribution, shape, and morphology were automatically selected by BESA ${ }^{\circledR}$ software. We used a $75 \%$ correlation cut-off between the search and target patterns with a reasonable range from 60 to $90 \%$. To clearly identify focal spikes emerging from the noisy EEG background signals, a bandwidth of $2-70 \mathrm{~Hz}$ was applied.

EEG "IES epochs" were defined to the last $1 \mathrm{~s}$ before and after the IES peak (T0). Non-overlapping IES epochs lasting $2 \mathrm{~s}$ (comprising 2,048 data points) were considered for each spike set, to allow sufficient surrounding background (baseline) activity for analysis.

Single IES epochs were inspected for artifact contamination. Individual rejection criteria were based on the distribution of IES epochs in terms of mean amplitude and gradient (first temporal derivative) values. IES Epochs that were contaminated with artifacts after visual correction, representing a total of $15 \%$, were rejected. Only IES epochs with background amplitude less than $200 \mu \mathrm{V}$ were considered. Artifact-free IES epochs were then submitted to the source localization and time-frequency spectral analysis steps described below.

At the end of the marking process, the selected IES epochs were overlapped in a single diagram to confirm that they presented similar shapes. Finally, all steps of IES selection and artifact rejection were manually reviewed by two experienced neurophysiologists (FW, EB).

A mean of about 100 spikes per patient were finally analyzed.

\section{Interictal Spike Source Localization}

To assess the homogeneity of the BECTS population, the sources of the average IES were estimated. To allow further analysis, the sources of IES must be located, for all patients, along the central sulcus with a tangential orientation and an anterior positivity of the dipole (Ishitobi et al., 2005). Due to the lack of a standardized validation method across source localization methods in clinical studies and considering the intrinsic limitation of each source modeling, different approaches may help clarify the nature of the presumed cortical source better than one approach can alone. Dipole and distributed EEG source localization are complementary and both methods are widely used to localize BECTS activity (Kamada et al., 1997; Lin et al., 2003; Huiskamp et al., 2004; Ishitobi et al., 2005; Pataraia et al., 2008; Kakisaka et al., 2011). Therefore, in our study, in addition to dipole based model (Dipole fitting), we used an algorithm named "Standardized Shrinking LORETA-FOCUSS" (SSLOFO) that integrates the two techniques (i.e., sLORETA and FOCUSS).

SSLOFO is an approach to combine the advantages of both low- and high-resolution methods in an automated fashion. Starting from a very smooth estimate, SSLOFO improves the spatial resolution using the recursive strategy of FOCUSS.

Artifact-free IES epochs were averaged and filtered with a $1 \mathrm{~Hz}$ high-pass filter (high pass: $6 \mathrm{~dB}$ /octave, zero phase) (Herrendorf et al., 2000; Ochi et al., 2000; Lantz et al., 2003; Groening et al., 2009; Elshoff et al., 2012). A standardized finite element head model (FEM) created from an averaged head of 50 individual MRIs in Talairach space (BESA Research ${ }^{\circledR}$ template), was used. This head model provided a realistic approximation of three compartments (brain, skull, and scalp) and was applied with the conductivity parameters of Scalp $0.33 \mathrm{~S} / \mathrm{m}$, Skull $0.0042 \mathrm{~S} / \mathrm{m}$, and Brain $0.33 \mathrm{~S} / \mathrm{m}$.

\section{Time-Frequency Analysis}

To characterize more precisely changes in neuronal activity occurring during each selected epoch, time-frequency analysis was performed, for frequencies between 4 and $200 \mathrm{~Hz}$ and including phase-locked and non-phase-locked representations to the IES.

To accomplish this goal, analysis were performed in 3 steps (pre-processing, time frequency representation, and statistical analysis) described in the following outlines.

\section{Pre-processing}

Non-overlapping IES epochs lasting $2000 \mathrm{~ms}$ were considered for each IES. A first relative baseline segment lasting $400 \mathrm{~ms}(-1000$ to $-600 \mathrm{~ms}$ before $\mathrm{T} 0$ ) was defined for each channel on each IES 
epoch. Time-frequency analysis was performed on the window between -1000 and $+1000 \mathrm{~ms}$ around $\mathrm{T} 0$.

To take into account a possible baseline selection effect, a second analysis was performed for 2 patients (patients 2 and 4) considering a larger and more distant reference period $(-3000$ to $-1000 \mathrm{~ms}$ before $\mathrm{T} 0$ ). Time-frequency analysis was performed on the window between -3000 and $+1000 \mathrm{~ms}$ around $\mathrm{T} 0$.

During the time-frequency averaging process, IES epochs were averaged without filtering to maintain the full bandwidth for time-frequency processing.

\section{Time-frequency representation (TFR) (Figure 1)}

TFR was performed according to the procedures described by Hoechstetter et al. (2004) and implemented in BESA Research ${ }^{\circledR}$. This procedure is able to distinguish global representations including phase-locked and non-phase-locked representations and extract induced representations corresponding to nonphase-locked activity (Figure 1).

\section{Global Time-Frequency Representation (GTFR):}

TFRs were first computed on each selected IES epoch at each frequency. This method precisely identified the nesting principles that specifically underlie the IES activity. To establish the regional specificity of these findings, TFRs were extended to include all EEG channels.

TFRs were computed by applying complex demodulation (Papp and Ktonas, 1977). For each frequency of interest $f_{0}$, the following three steps were performed:

1) The original time-domain signal (i.e., not subjected to any offline filtering) was multiplied by $\sin \left(2 \pi f_{0} f\right)$ and $\cos \left(2 \pi f_{0} f\right)$, respectively. This modulation operation shifts every signal at frequency $f$ to the difference and sum frequencies $\left(f \pm f_{0}\right)$ in the frequency domain.

2) The resulting two signals were low-pass filtered to extract the frequency range originally centered around $f_{0}$ and that was shifted to the low frequency range $\left(f-f_{0}\right)$. Thus, the low-pass cut-off frequency sets half of the width of the frequency band for which the envelope amplitude and phase is computed.

3) The two output signals of step (2) define the real and imaginary part of a complex signal as a function of time. Its magnitude corresponds to half of the envelope amplitude.
The time-frequency representation was calculated over each IES epoch. Frequencies were sampled (Gaussian filter) in $2 \mathrm{~Hz}$ steps and latencies were sampled in $25 \mathrm{~ms}$ steps, corresponding to a time-frequency resolution of $\pm 2.83 \mathrm{~Hz}$ and $\pm 39.4 \mathrm{~ms}$ at each time-frequency bin (full width at half maximum).

The TFRs of EEG activity was compared to the baseline segment, lasting $400 \mathrm{~ms}$ ( -1000 to $-600 \mathrm{~ms}$ before T0 of each IES) for all the patients and to the second baseline $(-3000 \mathrm{~ms}$ to $-1000 \mathrm{~ms}$ before T0 of each IES) for the patients 2 and 4 . Because the amplitude of human surface EEG waves is in the range of 10 to $100 \mu \mathrm{V}$ (Tong and Thakor, 2009), the averaged power of EEG baseline ongoing activity period $\left[\mathrm{P}_{\text {baseline }}(\mathrm{f})\right.$ below $]$ over about 100 IES epochs never goes to zero or near zero. Also, TFRs was expressed as the relative power change to baseline activity at a time-frequency bin compared with the mean power over the baseline epoch for that frequency, $T F R=\frac{\mathrm{P}(\mathrm{t}, \mathrm{f})-\mathrm{P}_{\text {baseline }}(\mathrm{f})}{\mathrm{P}_{\text {baseline }}(\mathrm{f})} \cdot 100$ where $P(t, f)=$ power at time $t$ and frequency $f$ and $\mathrm{P}_{\text {baseline }}(\mathrm{f})=$ mean activity at frequency $f$ over the baseline epoch.

This procedure yields TFRs containing phase-locked as well as non-phase-locked responses.

\section{Induced Time-Frequency Representation (ITFR):}

In studying oscillatory epileptic spike, it is possible that any change in oscillatory activity (especially higher frequency) that is related to an IES is time-locked to this IES but not necessarily phase-locked. The reason is that oscillations are ongoing phenomena that also exist in the absence of any IES. As a result, the phase of the oscillation at the time of occurrence of an IES is variable. In order to specifically assess the non-phase locked activity of IES (called induced activity), we subtracted the time-frequency representation of patient's averaged trials from the Global Time-Frequency Representation to create averages of the non-phase-locked spectral power only (see Figure 1 for detailed analysis block diagram).

\section{Statistical Analysis}

The probability that a power differs significantly from the average power during the baseline interval was investigated. Two-sided bootstrap testing were performed on the trials (Davidson, 1999). $\mathrm{z} 0$ and the test statistics $\mathrm{z}^{*}$ for a given bootstrap sample were

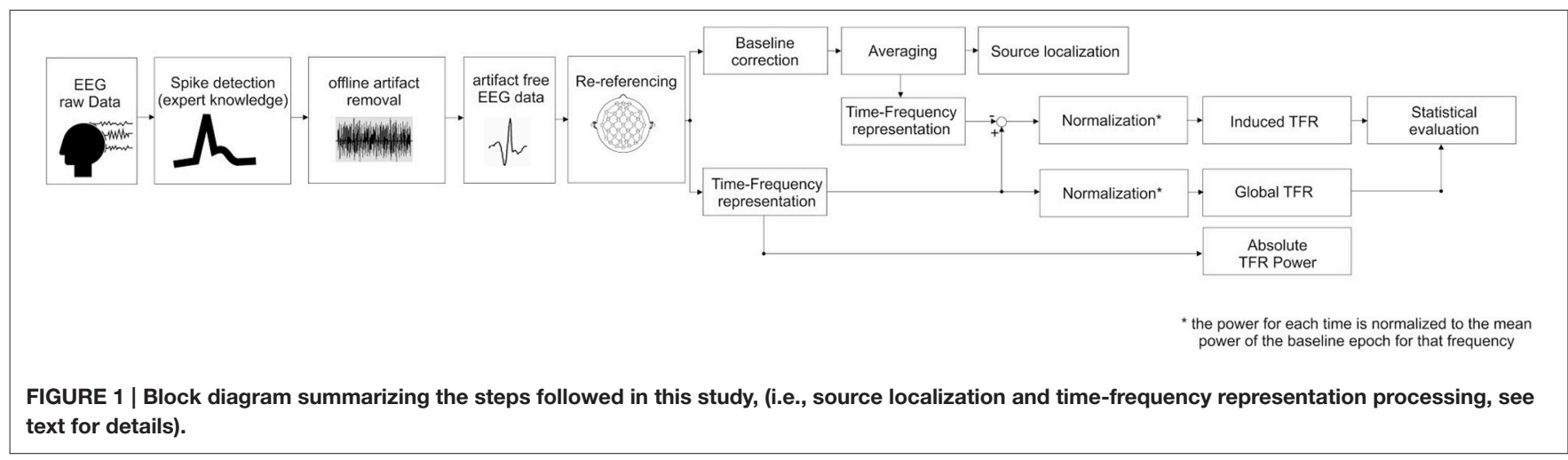


computed as

$$
z 0=\frac{\bar{y}_{2}-\bar{y}_{1}}{\sqrt{\frac{\sigma_{y_{2}}^{2}}{n}+\frac{\sigma_{y_{2}}^{2}}{n}}}, z^{*}=\frac{\bar{y}_{2}^{*}-\bar{y}_{1}^{*}-\left(\bar{y}_{2}-\bar{y}_{1}\right)}{\sqrt{\frac{\sigma_{y_{2}}^{2}}{n}+\frac{\sigma_{y_{2}}^{2}}{n}}}
$$

Where,

$$
\begin{aligned}
& \bar{y}_{1}=\frac{1}{n} \sum_{\text {trials }} \bar{P}_{\text {baseline }, i} \\
& \bar{y}_{2}=\frac{1}{n} \sum_{\text {trials }} P_{i}
\end{aligned}
$$

Here $\mathrm{P}$ denotes the power, $\mathrm{n}$ the number of trials. An asterisk denotes the value of a bootstrap sample. $\mathrm{R}$ bootstrap samples were computed. This computation was performed for each sampling point in time-frequency space. The $p$ value was approximated from the number of bootstrap samples where $\mathrm{z}^{* 2}$ $>z_{0}^{2}: p=\left(1+\#\left\{z^{* 2}>z_{0}^{2}\right\}\right) /(R+1)$.

To reduce the false positive rate (FPR), correction for multiple testing was performed using the method of Simes (1986). It is applied to each IES epoch which belongs to one frequency bin. This means that each channel and each frequency bin was treated as an independent measurement, whereas the statistical tests over the time series within one frequency bin were treated as multiple measurements. This approach was suggested by Auranen (2002). The reasoning behind it is that, it is difficult to define a rule for dependencies between channels. Each montage and each physiological phenomenon leads to different dependencies between the measurement channels. It was assumed that the activity which we were interested in is based on oscillatory phenomena, which were likely to be confounded to defined frequency bands. To assess the strength of the observed effects and for FPR correction, all $p$ values of one frequency bin and channel were sorted in ascending order $\left(\mathrm{p}_{i}, i=1, \ldots, N\right)$. The maximum index $m$ in the sorted array for which $\mathrm{p}_{i}<\alpha^{*} \mathrm{i} / \mathrm{N}$ was determined. All values with $i<m$ were accepted as significant detection. The significance level $\alpha$ is set to 0.05 .

\section{Control Conditions \\ Random triggers}

To reach the specificity of IES time-frequency representations, all the same 3-step procedure of time frequency analysis was performed with control random triggers.

Control random segments $(n=197)$, lasting $2000 \mathrm{~ms}$ around the random triggers, were analyzed. They were selected during similar period and background activities during which the effects of IES were analyzed. The $1000 \mathrm{~ms}$ before each EEG epoch and the epoch (lasting $2000 \mathrm{~ms}$ ) did not contain any IES. T0 was defined by random trigger time which corresponds to the central point of the epoch $(-1000 \mathrm{~ms},+1000 \mathrm{~ms})$.

The TFR procedure described above was performed using the baseline segments lasting $400 \mathrm{~ms}$ ( -1000 to $-600 \mathrm{~ms}$ before T0)."

\section{BECTS spikes simulation}

In order to reproduce the BECTS EEG data, one equivalent current dipole was fitted, located in the left central sulcus and characterized by an oblique orientation toward the mid-line, EEG data was generated using a spherical four-shell head model (Berg and Scherg, 1994; BESA ${ }^{\circledR}$ Dipole Simulator) with the following parameters.

- Radius of the head model $85 \mathrm{~mm}$

- Thickness of layers (mm):
- Scalp $6 \mathrm{~mm}$
- Bone $7 \mathrm{~mm}$
- CSF $1 \mathrm{~mm}$
- Conductivities (mho/m):
○ Scalp 0.33
○ Bone 0.0042
- CSF 1.0
○ Brain 0.33

All the TFR procedure has been applied to the simulated IES.

\section{RESULTS}

Eight male patients were included in the study. On HD EEG recording (64 electrodes), unilateral IES were selected in 6 patients. They were bilateral and independent in 2 other patients. This resulted in a total of 10 groups of IES.

\section{Clinical Data (Table 1)}

According to the inclusion criteria, all patients presented sensori-motor seizures with or without secondary generalization, regardless of whether IES were unilateral or bilateral. Five patients were taking antiepileptic drugs at the time of the recording. Two patients were not seizure-free. Four children had attention disorder and language impairment with no global deficit on the Wechsler Intelligence Scale for Children (WISC) IV test.

\section{Source Localization (Figure 2)}

The Source localization of the IES was performed in order to further assess the homogeneity of patients' population.

In all children, interictal source localization, using a dipole (Dipole fit) or distributed method (SSLOFO), confirmed the origin of the IES along the central sulcus (Ishitobi et al., 2005). The localization of the dipole at the first negative deflexion, and its tangential orientation with an anterior positivity was consistent with the expected precentral origin in BECTS patients (Ishitobi et al., 2005) (Figure 2). The patients' population was therefore considered to be clinically and electrophysiologically homogeneous, allowing further analysis.

\section{Time-Frequency Analysis}

The first step consisted of computing local changes in synchronization surrounding the IES for all frequency bands. Changes in synchronization occurring simultaneously around the IES, in areas distant to the epileptogenic zone were then analyzed. 
TABLE 1 | Clinical data for the 8 patients with BECTS.

\begin{tabular}{|c|c|c|c|c|c|c|}
\hline Patients & $\begin{array}{l}\text { Age at diagnosis } \\
\text { of BECTS (years) }\end{array}$ & $\begin{array}{l}\text { Clinical features } \\
\text { of seizures }\end{array}$ & Seizure-free* & $\begin{array}{l}\text { Antiepileptic } \\
\text { drugs }\end{array}$ & Standard EEG & $\begin{array}{l}\text { Neuropsychological } \\
\text { data }\end{array}$ \\
\hline 1 & 5 & GTCs & YES & - & Left CTs & Attention deficit \\
\hline 2 & 5 & GTCs & YES & VPS & Right CTs & $\begin{array}{l}\text { Attention deficit, } \\
\text { Language impairment }\end{array}$ \\
\hline 3 & 7 & PS & YES & - & Right and left CTs & Normal \\
\hline 4 & 9 & PS & $\mathrm{NO}$ & - & Right CTs & Normal \\
\hline 5 & 8 & PS & YES & LVM-VPS & Left CTs & Normal \\
\hline 6 & 9 & PS & YES & VP & Right CTs & Attention deficit \\
\hline 7 & 5 & PS & $\mathrm{NO}$ & VPS-OXC & Right \& left CTs & Attention deficit \\
\hline 8 & 6 & PS & YES & VPS-OXC & Left CTs & Normal \\
\hline
\end{tabular}

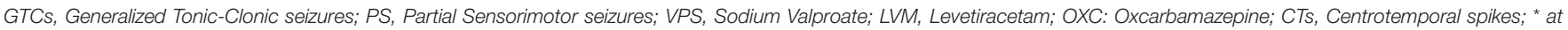
the time of HD EEG.

\section{Local Synchronization Changes (Table 2, Figures 3, 4)}

For all groups of IES, time-frequency analysis demonstrated statistically significant changes $(p<0.0002)( \pm 400 \mathrm{~ms}$ around the IES) compared to the 2 reference periods $(-600,-1000 \mathrm{~ms}$ and $-3000,-1000 \mathrm{~ms}$ before IES), for both $\mathrm{G}_{\mathrm{TFR}}$ and $\mathrm{I}_{\mathrm{TFR}}$.

\section{Hypersynchronization occurring simultaneously with the IES (see Table 2, Figure 3)}

Independently of the baseline considered, signal power increased significantly ( $p_{\text {corrected }}<0.0002$ ) in $\mathrm{G}_{\mathrm{TFR}}$ between 4 and 50 $\mathrm{Hz}$ (Figure 3). This hypersynchronization involved $88 \%$ of electrodes [45 to 64 (70-100\%) electrodes] (Table 2). Similar results were observed for $\mathrm{I}_{\mathrm{TFR}}\left(p_{\text {corrected }}<0.0002\right)$ (Figure 3), but with a narrower spatial extension around the epileptogenic zone, with only $53 \%$ of electrodes involved [8 to $64(12-100 \%)$ electrodes] (Table 2).

At frequencies higher than $50 \mathrm{~Hz}$, a significant $\left(p_{\text {corrected }}<\right.$ 0.0002 ) increase in signal power was observed for 6 of the 10 groups of IES. The spatial extension included 32\% (1 to 39 electrodes) and 28\% (1 to 39 electrodes) of the electrodes in $\mathrm{G}_{\mathrm{TFR}}$ and $\mathrm{I}_{\mathrm{TFR}}$ respectively (Table 2). These significant high-frequency synchronizations occurred concomitantly and continuously with hypersynchronization of lower frequencies.

\section{Time-frequency changes surrounding the IES (Table 2, \\ Figure 4)}

Independently of the baseline considered, $\mathrm{G}_{\mathrm{TFR}}$ were significantly modified, between 4 and $50 \mathrm{~Hz}$, in the -400 to $+400 \mathrm{~ms}$ window around the hypersynchronization of the IES (T0) for all groups of IES. No changes surrounding T0 were observed in the frequency domain higher than $50 \mathrm{~Hz}$.

Two different patterns of synchronization changes were observed in the time-frequency domain.

Pattern 1: For 6 of the 10 groups of IES, time-frequency changes consisted of a significant $(p<0.0002)$ decrease in the power of frequencies below $50 \mathrm{~Hz}$ before $(-400,-100$ $\mathrm{ms})$ and after $(+100,+400 \mathrm{~ms})$ the IES, likely corresponding to a decrease in synchronization preceding and following hypersynchronization of the IES, i.e., a kind of mirror desynchronization surrounding the IES (Figure 4). In GTFR, this desynchronization involved $37 \%$ of electrodes (10 to $95 \%$ of electrodes). Similar results were observed for $\mathrm{I}_{\mathrm{TFR}}$, but over a more limited area (26\% of electrodes, 3-84\%) adjacent to the epileptogenic zone (Table 2 ).

Pattern 2: Instead of the desynchronization described in pattern 1 , a significant $(p<0.0002)$ oscillation in hypersynchronization was observed in the same time window $(-400,+400 \mathrm{~ms})$ surrounding hypersynchronization of the IES $(-100,+100 \mathrm{~ms})$ for 4 of the 10 groups of IES (Figure 3 ). The spatial extension included $71 \%$ (42 to $100 \%$ ) and $66 \%$ (35 to $100 \%$ ) of electrodes in $\mathrm{G}_{\mathrm{TFR}}$ and $\mathrm{I}_{\mathrm{TFR}}$, respectively (Table 2).

It should be stressed that neither pattern 1 nor pattern 2 was correlated with any changes in HD EEG raw activity, including the slow waves preceding or following the IES (see Figure S2).

TFRs were not affected by the use of different baselines (Figure 4).

\section{Distant Synchronization Changes Surrounding the IES (Table 2, Figure 5)}

In 7 of the 10 groups of IES, a significant decrease $(p<$ 0.0002 ) in signal power was observed for $G_{T F R}$ and $I_{T F R}$ at frequencies below $10 \mathrm{~Hz}$ in areas distant from the IES onset zone. This distant desynchronization occurred during the same time window $(-400,+400 \mathrm{~ms})$ during which the synchronization power was either decreased (pattern 1) or oscillatory (pattern 2) in the epileptogenic zone (Table 2) (Figure 4). These desynchronizations in low-frequency bands occurred in frontal $(n=7)$ and/or temporal $(n=4)$ and/or occipital $(n=2)$ areas are highly suggestive of repetitive and transient desynchronizations distant from the epileptogenic zone of the IES.

Like for TFRs observed in the epileptogenic zone, TFRs in distant areas were not affected by the different baselines (Figure 4). 


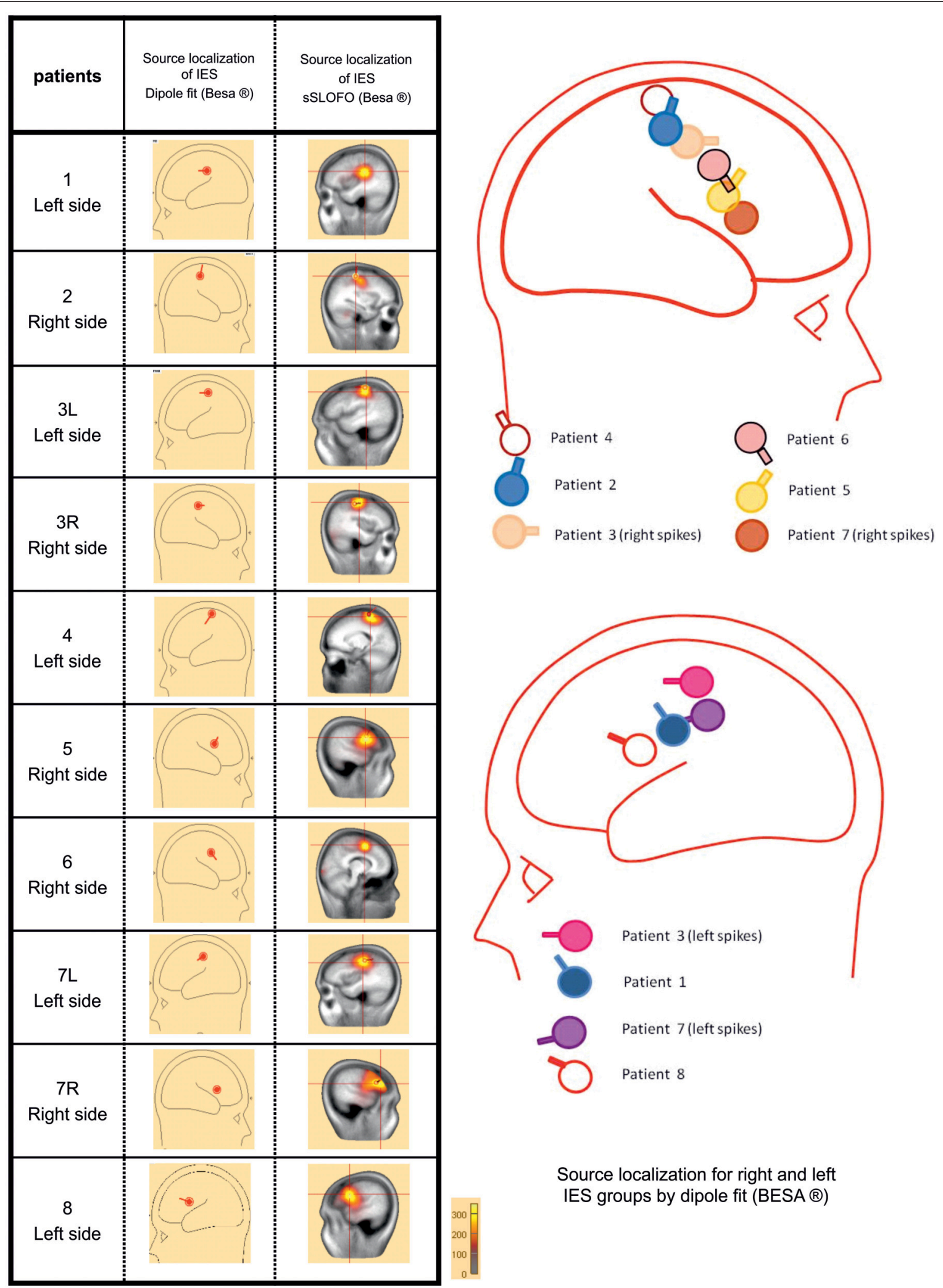

FIGURE 2 | Source localization using the dipole fitting method and the distributed methods for each group of IES. In all children, source localization, whatever the method used confirmed the origin of the IES, along the central sulcus with a tangential dipole orientation and an anterior positivity consistent with a precentral origin. 
TABLE 2 | Results of global and induced time-frequency responses for all IES groups, simultaneously with IES and locally or at a distance around IES

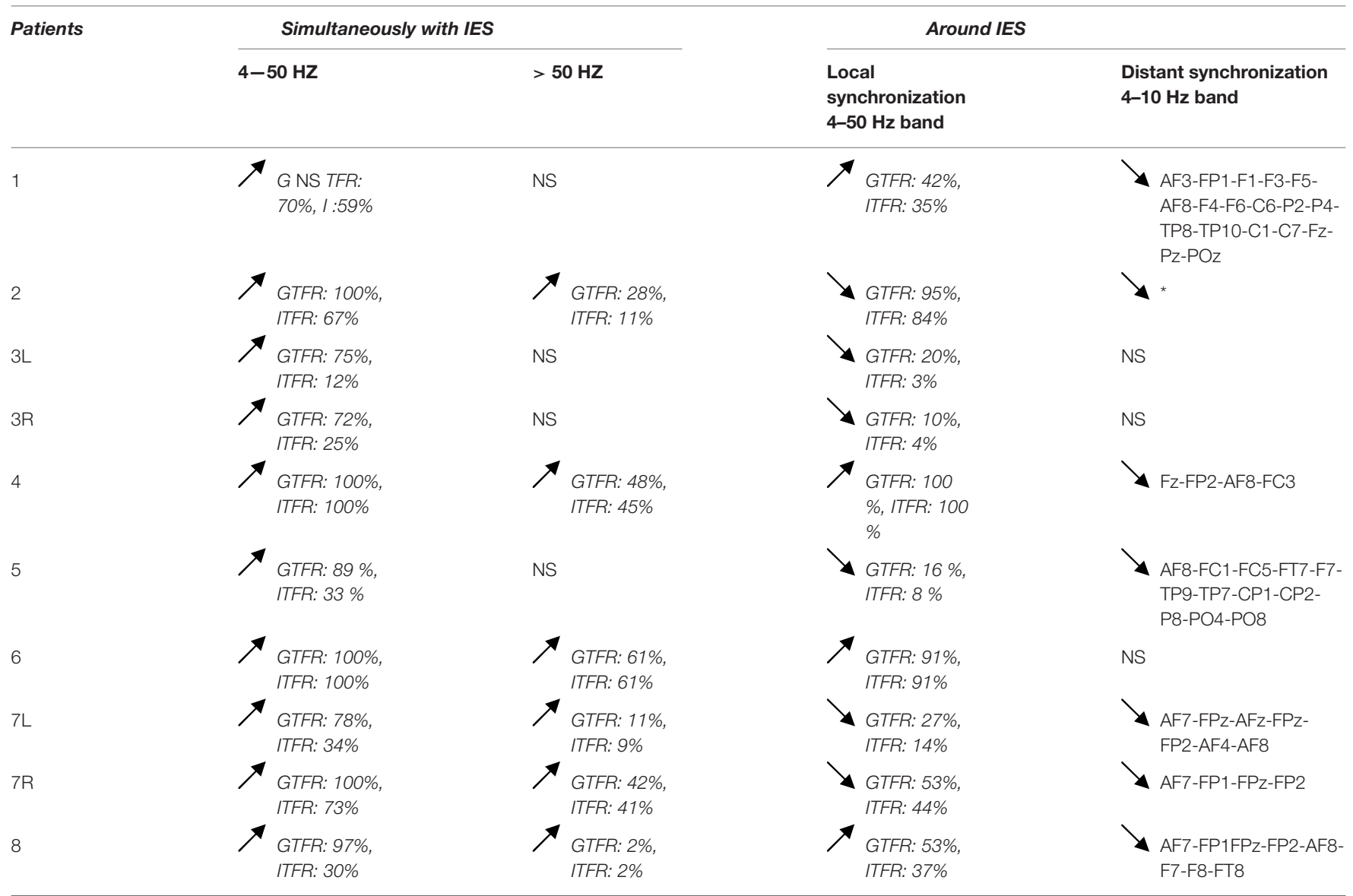

Increase of power signal analyzed by time-frequency method (baseline [-1000; -600 ms] $(p<0.0002)$

1 Decrease of power signal analyzed by time-frequency method (baseline [-1000 ms; -600 ms] ( $p<0.0002)$

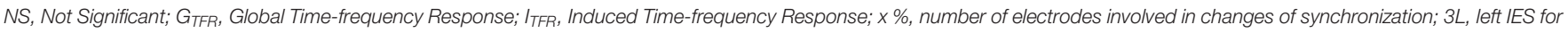
patient 3; 3R, right IES for patient 3; 7L, left IES for patient 7; 7R, right IES for patient 7.

* Electrodes involved in decreased distant synchronization for patient 2: AF3-AF7-FP1-F1-F3-F5-F7FC1-FC5-FC3-F17-C1-C3-C5-T7-CP1-Cp3-Cp5-TP7-TP9-P3-P5-P7-O1-PO3PO7-FPz-AFZ-FZ-FCZ-CZ-CPZ-Pz-POz-OZ-FP2-AF4-AF8-F2-F4-F6-F8-FC2-FC4-FC6-FT8-C6-T8- Cp6-TP8-P6-O2-PO4

\section{Time-Frequency Changes in Control Conditions (Figures 6, 7)}

In order to evaluate the specificity of low-frequency activity, exactly the same time-frequency analysis that we have done for the IES was performed but using the random triggers, out of IES. Time frequency analysis EEG activities related to the random trigger do not produce any statistically significant effects around the trigger, nor were hypersynchronization or desynchronization observed in either the epileptic zone or in the distant areas.

The TFR analysis performed on simulated IES returned isolated local hypersynchronization in $\mathrm{G}_{\mathrm{TFR}}$ in $4-30 \mathrm{~Hz}$ frequencies. Neither local nor distant changes surrounding IES were identified whatever the baseline period (Figures 5E,F, Figure S1).

\section{DISCUSSION}

Time-frequency analysis applied to HD EEG in a homogeneous population of male patients with BECTS demonstrated neuronal synchronization changes surrounding IES. Before and after the well-known hypersynchronization concomitant with the IES ( $\pm 400 \mathrm{~ms})$ there were: (i) two patterns of dysregulation in the epileptogenic zone and (ii) distant desynchronization, involving low-frequency bands in frontal, temporal, and occipital networks. These dysregulations might be involved in the mechanisms that propel neurons to synchronize and may play a role in the cognitive deficits observed in BECTS patients (Vannest et al., 2015).

\section{Methodological Considerations}

Because in BECTS, sources are modeled by one tangential source (Pataraia et al., 2008; Kakisaka et al., 2011), we analyse EEG potentials in the sensor space. The analysis in the sensor space might have led to overlap between the information of adjacent electrodes due to volume conduction effect which in turn may lead to spurious connectivity among neighboring channels. Surface Laplacian, might have been an 


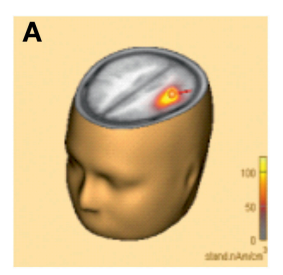

Source localization in $3 \mathrm{D}$ representation by dipole fit and distributed methods

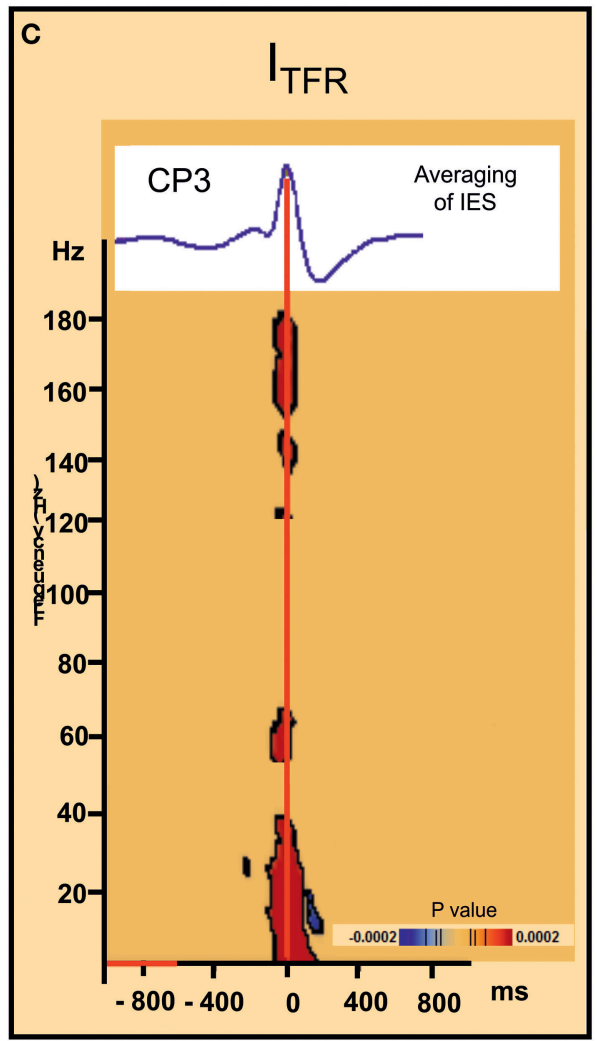

Reference period: [-1000 ms ; -600 ms]

\section{Patient 7, left IES}

\section{$\mathrm{G}_{\mathrm{TFR}}$}

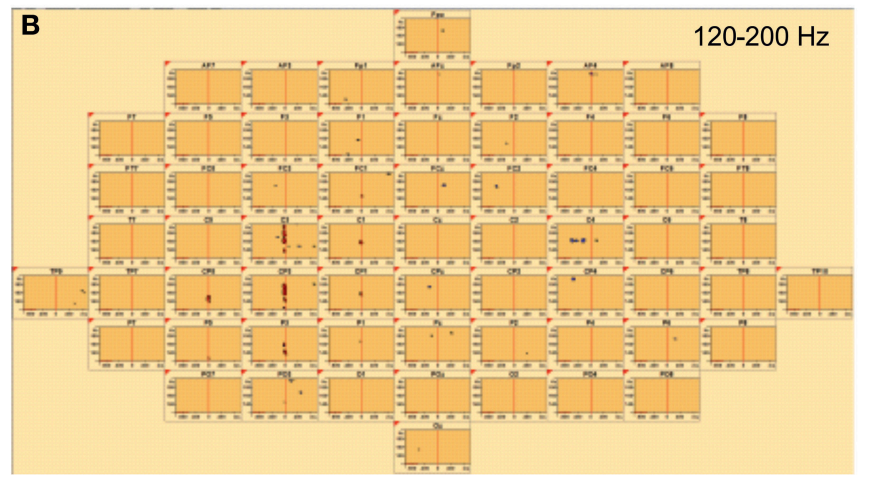

$50-120 \mathrm{~Hz}$
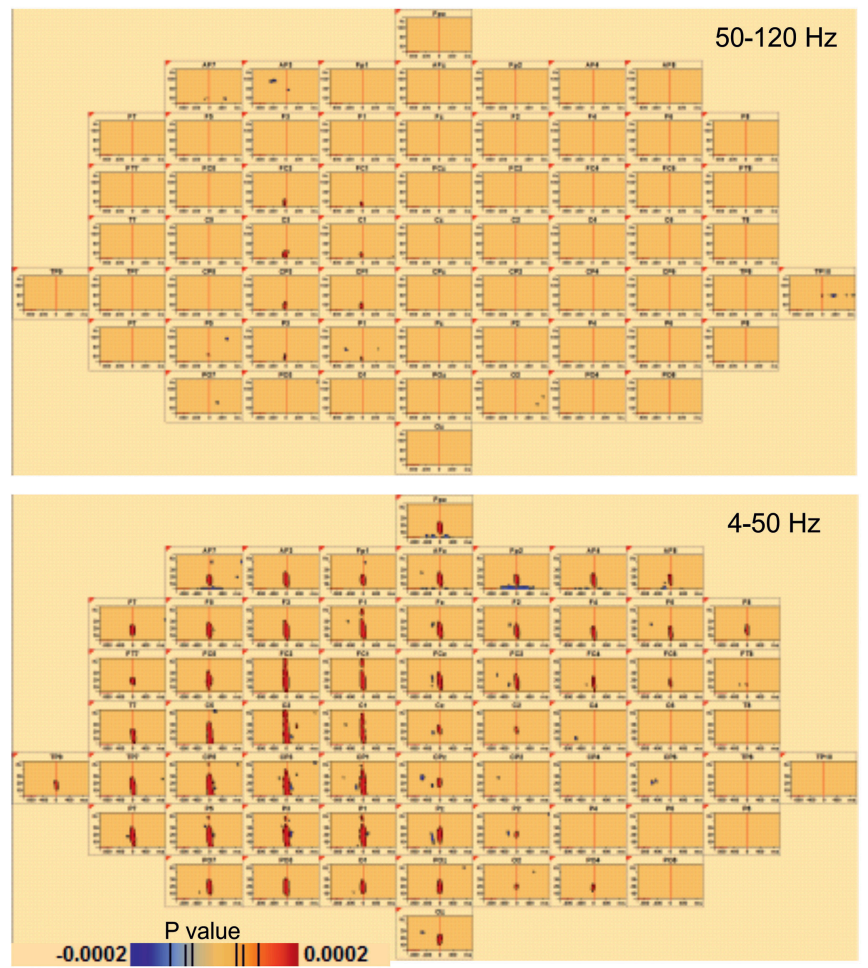

FIGURE 3 | Hypersynchronisation occurring simultaneously with the IES in the 4-200 Hz band in patient 7 (left IES) in Global (G responses (ITFR). (A) Source localization in dipole fit and SSLOFO in a 3D representation for the patient 7. (B) Significant Statistical results ( $p<0.0002)$ of time frequency analysis (induced activity) for frequencies between 4 and $50 \mathrm{~Hz}, 50$ and $120 \mathrm{~Hz}$, and 120-200 Hz. (C) On the top, result of the averaging of the IES for the patient 7. By time frequency analysis, Island of HFOs are simultaneously extracted with the IES, in induced activity (ITRF).

alternative since it have the effect of reducing the effective volume, thereby improving spatial resolution; as discussed in Nunez and Srinivasan (2006), potentials and surface Laplacians are sensitive to different spatial band-widths of the source distribution. Thus, surface Laplacians serve to complement (but not replace) EEG potentials (i.e., the surface Laplacian emphasizes certain types of source activity-More sensitive to radial than tangential dipoles, thus sources in sulci will be minimized-reduces the sensitivity of the EEG to sulci). In this paper we focused on the dynamics of neuronal networks surrounding IES which might improve our knowledge of the mechanisms that drive neurons to the hypersynchronization in BECTS. In our recently published connectivity study of IES in BECTS (Adebimpe et al., 2016), to identify the average location of interictal spike sources we used the exact Low Resolution Electromagnetic Tomography (eLORETA) method. Both approach returns similar results concerning the sources and the interactions of the IES with the frontal areas (Adebimpe et al., 2016)

Simultaneously with the IES, a significant broad band increase in the power spectrum was observed, including frequencies higher than $50 \mathrm{~Hz}$. 

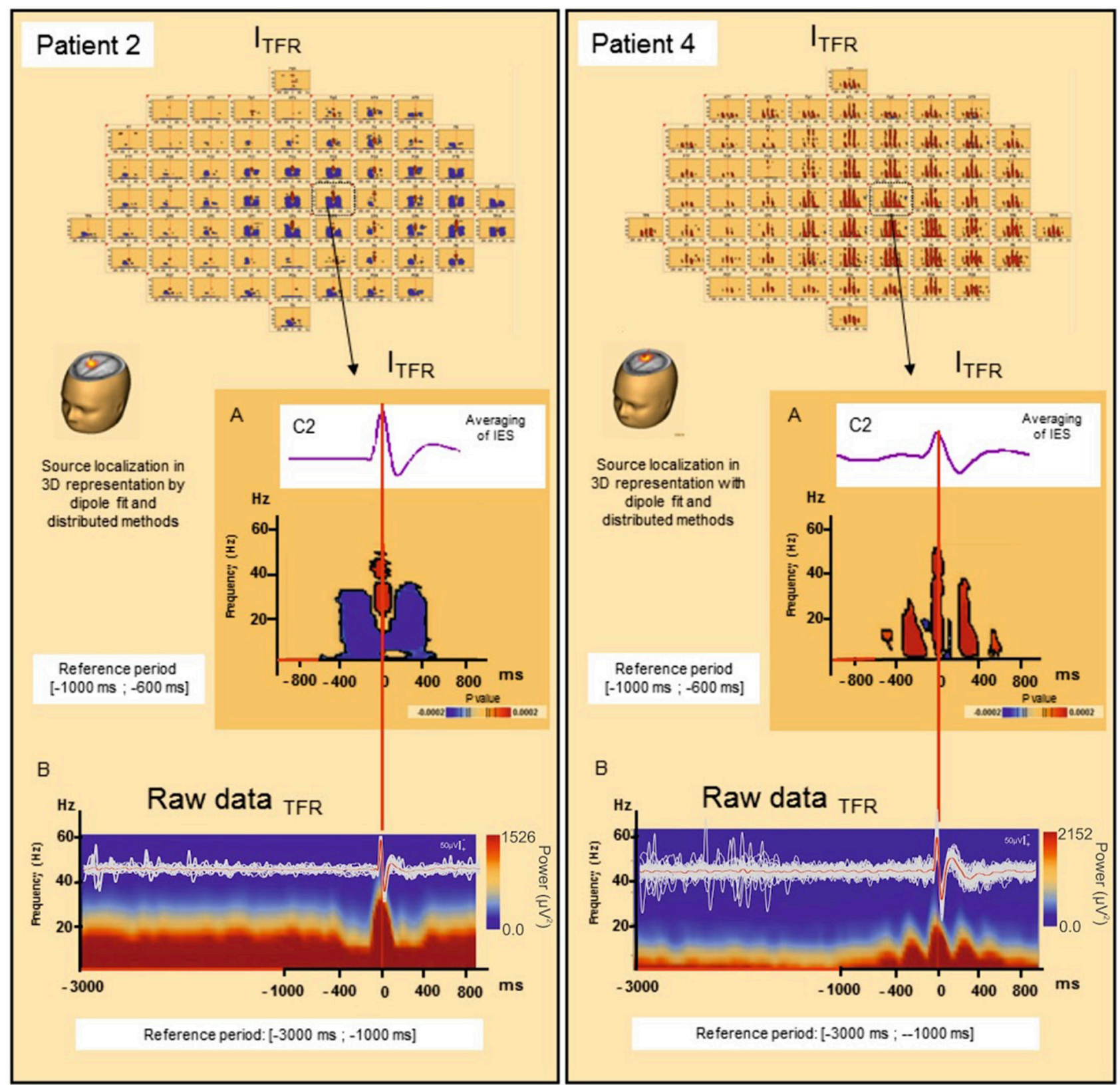

FIGURE 4 | Induced local changes in synchronization around the IES, examples of patients 2 and 4 . (A) On the top, results of the averaging of the selected IES. Bellow, Significant statistic $(p<0.0002)$ results of time frequency analysis in regard of the electrode $\mathrm{C} 2$ for patient 2 (on the left) and patient 4 (on the right) (reference period [ $-1000 ;-600 \mathrm{~ms}]$ ). (B) Raw data of time frequency analysis for the patient 2 (on the left) and 4 (on the right) (reference period: [ $-3000 ;-1000 \mathrm{~ms}]$ ). For patient 2, ITFR showed mirrored desynchronization around T0 for frequencies range from 4 to $50 \mathrm{~Hz}$ independently of the baseline considered [-1000; $-600 \mathrm{~ms}$ ] (A) or [-3000 ms; -1000 ms] (B). This desynchronization was localized nearly of the epileptogenic zone. For patient 4, a progressive increase in synchronization mirrored around the IES, in the same frequencies were found [baseline [-1000; $-600 \mathrm{~ms}$ (A) or [-3000 ms; $-1000 \mathrm{~ms}]$ (B)]. T0: define by the peak of the first negative deflexion of the IES.

To exclude the presence of false synchronizations caused by filtering of sharp transients (Bénar et al., 2010; Amiri et al., 2016), induced activities were extracted from global changes in synchronization. By means of this original approach, we showed that IES in BECTS are associated with a significant increase in high-frequency power $(50-200 \mathrm{~Hz})$, co-occurring with IES.
These increases could reflect, partially, high frequency oscillations (HFOs) widely recognized as a marker of epileptic tissue in partial refractory epilepsy, (Jacobs et al., 2011, 2012, 2016; Jefferys et al., 2012) but also observed in idiopathic partial epilepsies (Kobayashi et al., 2011; van Klink et al., 2016). 


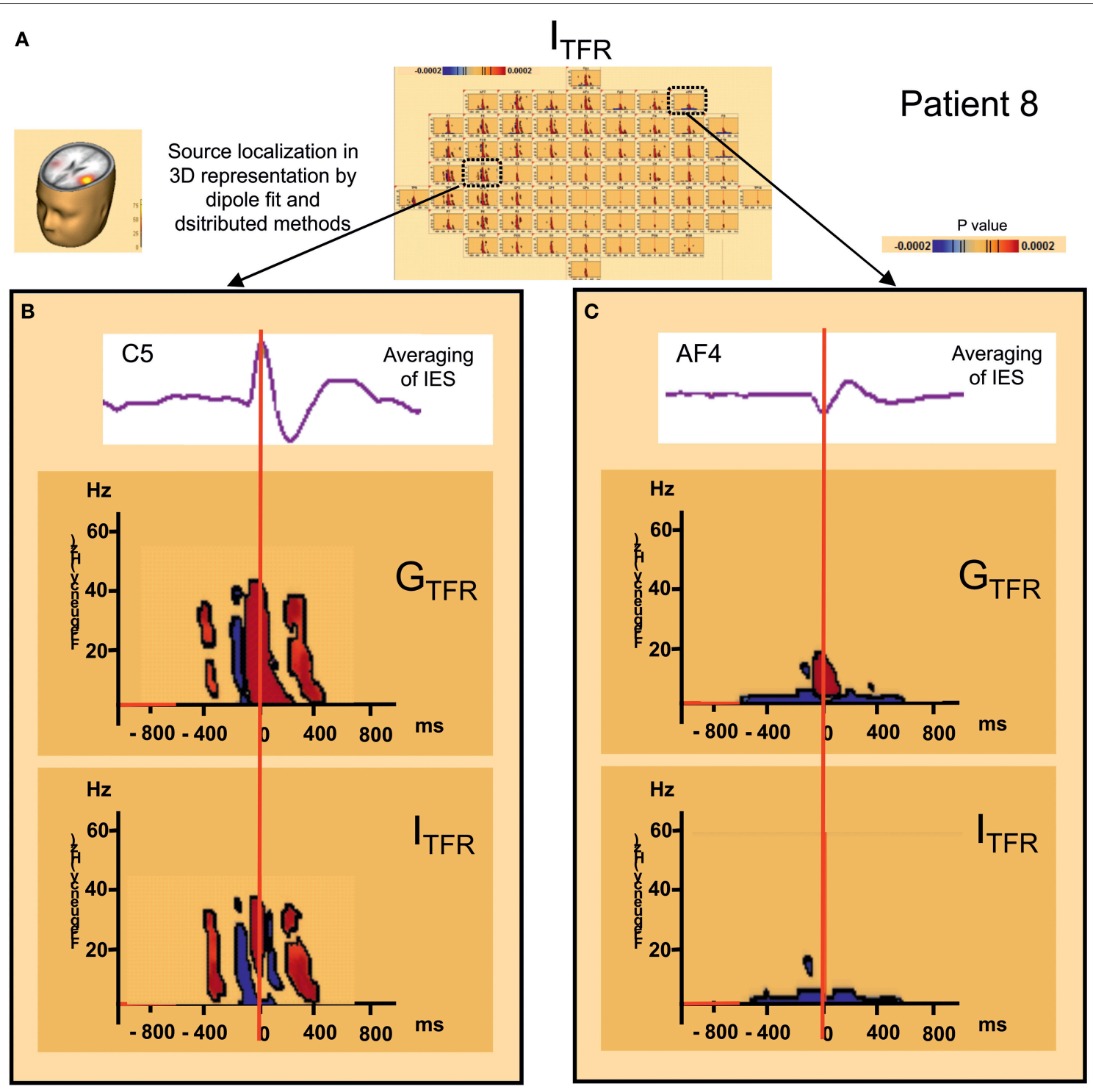

FIGURE 5 | Distant changes in synchronization (GTFR and ITFR) around IES. (A) Source localization and dipole fit and SSLOFO in a 3D representation for the patient 8. (B) Statistical significant results $(p<0.0002)$ of time frequency analysis [induced (IFR) and global (GTFR) time frequency results] in regard of the epileptogenic focus (electrode C5). (C) Statistical significant results $(p<0.0002)$ of time frequency analysis [induced (ITFR) and global (GTFR) time frequency results] in regard of the frontal area (electrode AF4). In ITFR and GTFR distant desynchronizations were observed, distant to the epileptogenic zone, notably in fronto-temporal areas, involving low frequency bands (bellow $10 \mathrm{~Hz}) \mathbf{( C )}$ in the same time window as local dysregulation of synchrony (B) $(\mathbf{F i g u r e} 3)[-400 \mathrm{~ms} ;+400 \mathrm{~ms}]$.

In our study, high frequencies power increases were more localized than the increases in lower frequencies, suggesting that they occurred in more restricted centro-temporal areas. Despite a potential volume conduction effect on the extension of HFO and their localization value, our results are in accordance with previous studies which showed, independently of the type of epilepsy, that spikes with HFOs are closely linked to the epileptogenic zone (Jacobs et al., 2009a). This remark also applied to the volume conduction effect on low frequency bands distant desynchronizations. They were not observed in the epileptogenic zone but only in restricted frontal areas and also unilateral. Altogether, this suggests that the volume conduction effect does not impact so much the localization in BECTS. However, the evaluation of the extension of the synchronization or desynchronization deserves further studies. One issue which could help to minimize this effect (Kayser and Tenke, 2006; Srinivasan et al., 2007) would be, to apply a spatial high-pass filter or other spatial transform such as currentsource-density, to reject zero-phase lag synchronizations (König et al., 1995; Rajagovindan and Ding, 2008; Vicente et al., 2008), to apply independent components analysis, which calculates unique generators of variance in the cortex (Makeig et al., 1997) or to estimate the cortical sources using beamforming (e.g., LCMV). 


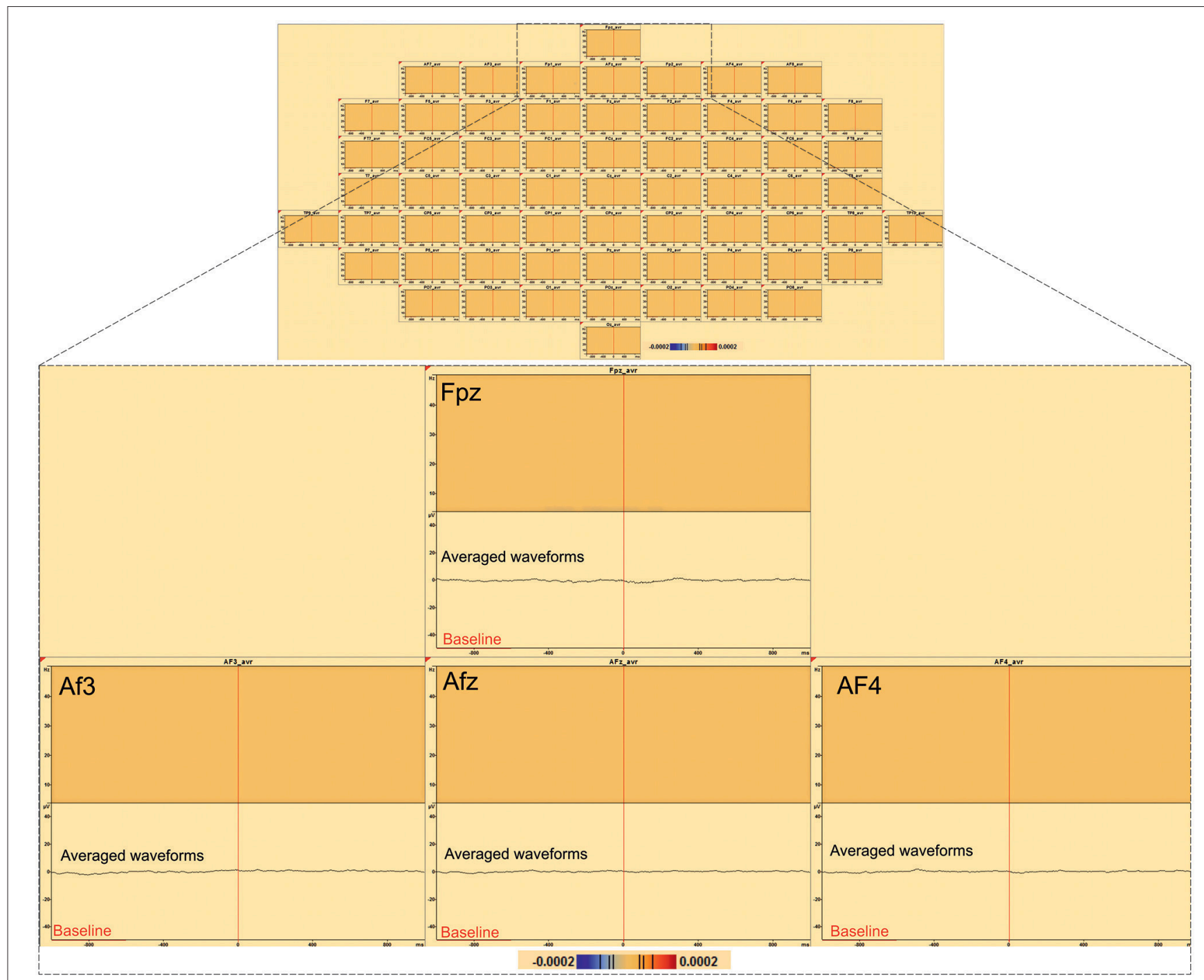

FIGURE 6 | Time-frequency analysis of 197 random triggers out of IES in patient $\mathbf{8}$. No significant statistical ( $p<0.0002$, corrected $p$-value) changes in random triggers out of IES were found by time frequency analysis (baseline [-1000; $-600 \mathrm{~ms}]$ ).

Hypersynchronization was preceded and followed by complex dysregulation of synchronization occurring within the epileptogenic zone and characterized by 2 specific patterns between 4 and $50 \mathrm{~Hz}$ : (i) a decrease in power frequencies and (ii) oscillation of power frequencies.

As this dysregulation consisted of 2 different patterns and was not affected by the use of different reference periods, it is unlikely to be due to a signal processing artifact or the resulting effect of a preceding masked IES not visualized on raw data. Moreover, Keller and collaborators found similar results in ECoG (Keller et al., 2010).

In the present study, we focused on changes in synchronization surrounding the IES, especially those changes preceding the IES. The references periods $(-1000 ;-600 \mathrm{~ms}$ or $-3000 ;-1000 \mathrm{~ms}$ ) therefore had to be selected in the same activation state, in the immediate temporal environment of the peak and away from the time window of interest $(-400 ;+400$ $\mathrm{ms}$ ). This requirement may explain some of the differences observed between our study and other published studies, in which a single reference period was chosen situated at a distance from the peaks (Jacobs et al., 2011) or constructed as representing an average brain activation state (Kobayashi et al., 2009). In these previous studies, the authors only identified desynchronizations after the IES (Kobayashi et al., 2009; Jacobs et al., 2011) and/or an inconstant hypersynchronization occurring before the IES that the authors characterized as HFOs (Kobayashi et al., 2009; Ren et al., 2015).

The mechanisms involved in these complex dysregulations have not yet been elucidated. They cannot be explained by synaptic interactions, gap junctions or ephaptic conduction involved in intrinsic membrane oscillations and responsible for the generation of HFOs (Jefferys et al., 2012), as they started 


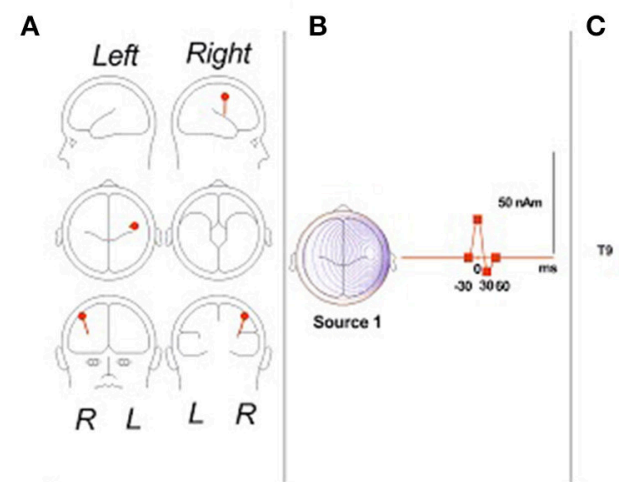

C

D
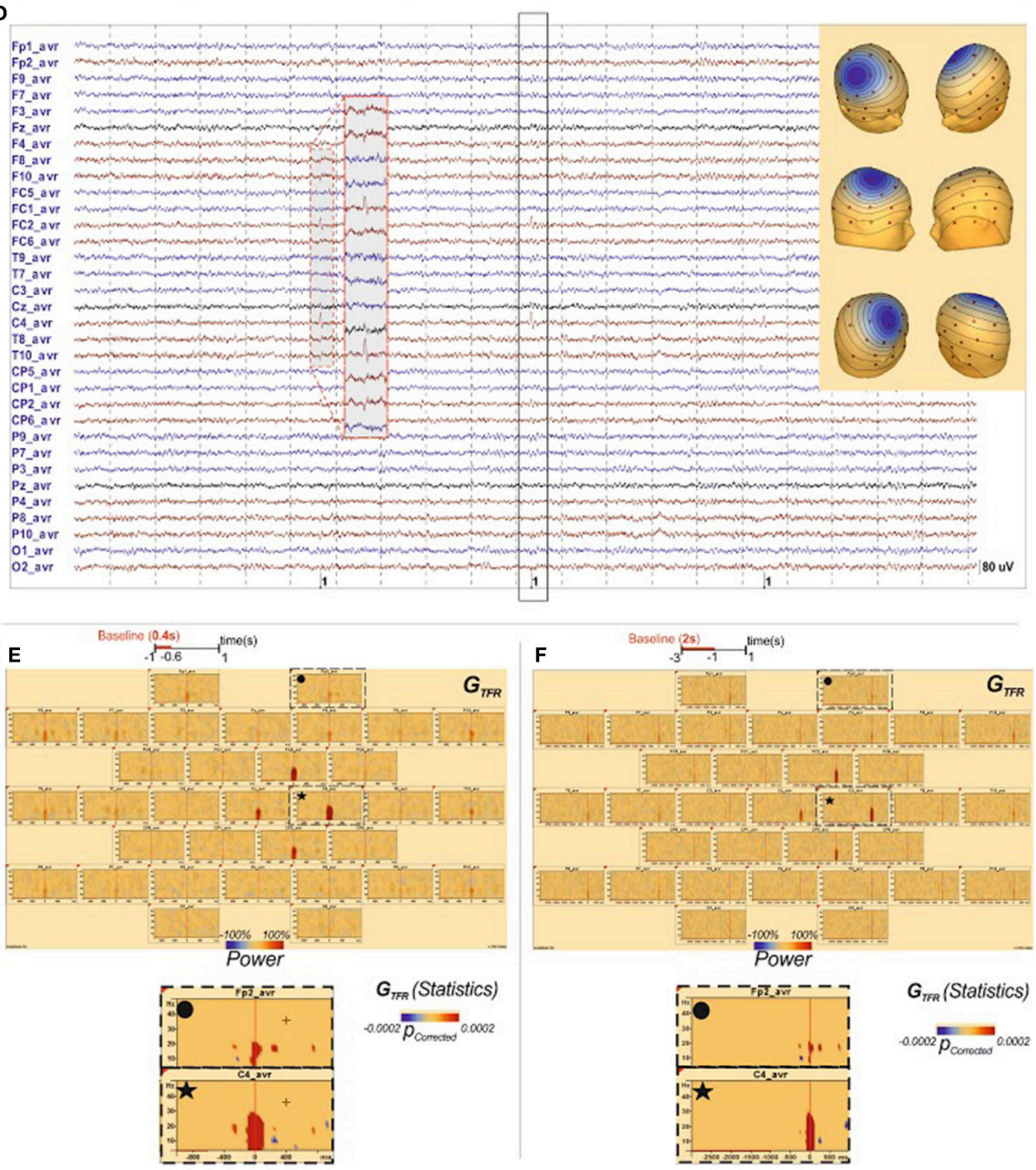

$G_{\text {IFR }}$ (Statistics)

$.0002 \bar{p}_{\text {Concosos }} 0.0002$

FIGURE 7 | Time-frequency analysis of IES simulation. (A) The source localization by dipole fit method identified an origin of the simulated IES in the right central sulcus, like described in BECTS (Ishitobi et al., 2005). Locations, orientations (A) and wave shapes (B) of the 1 dipole source, "the model sources," used to simulate 


\section{FIGURE $7 \mid$ Continued}

the EEG data. TO is denoted by the vertical mark on the time base. (B) These topographies reproduced properties expected for the scalp-recorded IES field, including central negativities, with corresponding positivities at lateral sites. (C) The simulated scalp IES in average reference format. The solid lines indicate the noise-free wave forms generated by the dipole sources indicated in Panel A. (C) indicate the signal + noise wave forms that were analyzed. (D) raw data of the simulated EEG and IES. Scalp potentials (33-channel montage) were simulated from dipoles (Berg, 2006) in order to correspond to field produced by the generator within right central sulcus. (E): GTFR of the simulated IES results with the [-1000 ms; $-600 \mathrm{~ms}$ ] baseline period: GTFR of the simulated IES demonstrated significant changes between 4 and $30 \mathrm{~Hz}$ in the right central sulcus simultaneously with the IES but no change around the IES in regard of the epileptogenic zone or in distant area. (F): GTFR of the simulated IES results with the [-1000 ms; $-600 \mathrm{~ms}$ ] baseline period: Like for the [-1000 ms; $-600 \mathrm{~ms}]$ baseline period, GTFR of the simulated IES demonstrated significant changes between 4 and $30 \mathrm{~Hz}$ in the right central sulcus simultaneously with the IES but no change around the IES in regard of the epileptogenic zone or in distant area.

only a few tenths of milliseconds before synchronization of the IES. Similarly, mechanisms proposed for the emergence of the IES are unlikely to contribute to the observed dysregulations. Although the Potential Depolarization Shift (PDS), the hallmark of the IES (Ayala, 1983), is much longer than the depolarization observed with normal excitatory postsynaptic potentials, the progressive recruitment of excitatory inputs that trigger the IES start only several tenths of milliseconds before onset of the IES, which does not correspond to the time window of $400 \mathrm{~ms}$ observed in our study. Other mechanisms, such as gap junction and calcium waves, notably involved in the initiation and propagation of synchronization to neighboring neurons, have been reported to be triggered simultaneously with the IES (Jefferys et al., 2012). Finally, the hyperpolarization following PDS, corresponding to the slow component of the spike wave discharge in EEG (Ayala, 1983; Neckelmann et al., 2000) cannot explain the desynchronization observed after the IES, as, in line with previous reports, desynchronization was observed regardless of whether or not the slow wave was present and therefore does not necessarily reflect the degree of post-spike depression (Jacobs et al., 2011).

It may be more relevant to consider our results at a mesoscopic/macroscopic level. The dynamics of the assembly of neurons in the epileptic network involved in the emergence of the IES are more complex, more heterogeneous and more variable than initially thought. Using multi-unit activity analysis in refractory epilepsy, Keller and collaborators observed a marked variability of cellular activation pattern (decrease or increase of neuronal activity) within the seizure onset zone, concomitantly to the IES (Keller et al., 2010). Moreover, activity changes were observed for some clusters of neurons at longer interval (400 ms) before the IES (Keller et al., 2010; AlvaradoRojas et al., 2013). Similarly, using the Fast Optical Signal (FOS) technique synchronized with ECoG in epileptic rats, we have shown changes in cellular conformation in the same time range (Manoochehri et al., 2017) suggesting cellular activations occurring well-before the IES. The two types of dysregulation observed in the present study are in line with these results and represent, at a macroscopic level, the variability of the strategy of neuronal assemblies to reach the freezing point beyond which recruitment of synaptic inputs will trigger the regenerative currents of the PDS and IES (Keller et al., 2010; Alvarado-Rojas et al., 2013; Manoochehri et al., 2017). Similarly, using a graphtheoretical approach, increases in network clustering around the IES, in both symptomatic partial epilepsy (Ibrahim et al.,
2014) and BECTS (Adebimpe et al., 2015a,b) suggest a more complex reorganization of the network in the epileptogenic zone, which can result in changes in the synchronization dynamics, as monitored by time-frequency analysis of scalp HD EEG.

\section{Desynchronization in Low-Frequency Bands, Distant to the Epileptogenic Zone}

In parallel to local desynchronization, our study demonstrates desynchronization in low-frequency bands, distant to the epileptogenic zone.

Like local reorganization of the neuronal networks, distant inward interactions are also likely to modify the functional environment inside the epileptic zone in which IES are triggered and the functional connectivity of the epileptic zone toward other areas (Adebimpe et al., 2015a,b, 2016). By extrapolating from the features described in seizures, in which desynchronizations or hypersynchronizations are observed several minutes or hours before the seizures, the dysregulation of synchronization observed in the present study would modify the input complexity and functionality of epileptogenic brain regions, creating an idle population of neurons that may be more susceptible to recruitment into IES (Le Van Quyen et al., 2005; Aarabi et al., 2008). Similarly, the previously described hemodynamic changes starting several seconds before the IES (Jacobs et al., 2009b; Osharina et al., 2010) are likely to modify the functional environment of the network around the IES.

However, the effect observed in distant areas is highly specific to the interictal spikes and are only observed $\pm 500 \mathrm{~ms}$ around the peak of the spikes concomitantly to the alternation of desynchronization-synchronization-desynchronization observed in the epileptic zone. In line with these results, by combining the EEG source imaging and the time varying effective connectivity method, stronger directional connections from the epileptic zone to the frontal regions were observed during interictal spikes in BECTS patients (Adebimpe et al., 2016) suggesting that benign epileptic network may be disrupted by IES (Adebimpe et al., 2015a,b).

It has long been suspected that IES contribute to cognitive and behavioral deficits (15-30\% of BECTS children), but the underlying physiological mechanisms are still poorly understood (Vannest et al., 2015). In BECTS, IES are associated with transient cognitive impairment (TCI) (Aarts et al., 1984; Fonseca et al., 2007), which starts several hundreds of milliseconds before the spikes (Van Bogaert et al., 2012). Reorganization of the networks in the epileptic focus and in other functionally connected areas might participate in TCI (Verrotti et al., 2014). In support of 
this hypothesis, connectivity analysis using EEG or fMRI has demonstrated deactivation associated with IES in widespread cortical areas involved in cognitive processing, including the frontal, temporal and occipital cortex (Besenyei et al., 2012; Adebimpe et al., 2015a; Xiao et al., 2016), as well as areas involved in the Default Mode Network (DMN) (Cataldi et al., 2013; Fahoum et al., 2013; Adebimpe et al., 2015b). IES would therefore repetitively and transiently disrupt the functionality of different networks involved in cognitive processing (Besseling et al., 2013; Verrotti et al., 2014). Although our study does not allow a straight full demonstration of the relationship between distant desynchronization and cognitive disorders, the concomitancy of desynchronization in distant areas constitutes one possible substrate for the functional disruption and network reorganization leading to TCI. Further prospective studies including neuropsychological tests performed at the time of the EEG are mandatory to more specifically evaluate the potential underlying mechanisms explaining the cognitive consequences of IES. Our results, notably in distant areas, constitute just the beginning of unraveling the nature of cognitive deficits in this population.

\section{CONCLUSION}

In the present study, we show that large-scale network changes also precede the IES, supporting the concept that epileptic dynamics cannot be viewed in isolation, but must be interpreted in the context of a dynamic system of interregional communication within larger networks.

In the epileptogenic zone, complex changes both shortly preceding and following IES, illustrated the complexity of the underlying mechanisms of IES generation.

Changes in desynchronization are also observed in distant zones from the epileptogenic area both shortly before and after IES, suggesting that neuronal reorganization is under the influence of a larger embedded network, which may play a causal role in the expression of the IES.

In concordance with other studies, these findings observed in male patients with BECTS are likely to be generalizable across different gender or other underlying epileptogenic syndromes and locations of epileptic foci.

\section{REFERENCES}

Aarabi, A., Wallois, F., and Grebe, R. (2008). Does spatiotemporal synchronization of EEG change prior to absence seizures? Brain Res. 1188, 207-221. doi: 10.1016/j.brainres.2007.10.048

Aarts, J. H., Binnie, C. D., Smit, A. M., and Wilkins, A. J. (1984). Selective cognitive impairment during focal and generalized epileptiform EEG activity. Brain 107, 293-308.

Adebimpe, A., Aarabi, A., Bourel-Ponchel, E., Mahmoudzadeh, M., and Wallois, F. (2015a). EEG resting state analysis of cortical sources in patients with benign epilepsy with centrotemporal spikes. Neuroimage Clin. 9, 275-282. doi: 10.1016/j.nicl.2015. 08.014

Adebimpe, A., Aarabi, A., Bourel-Ponchel, E., Mahmoudzadeh, M., and Wallois, F. (2015b). Functional brain dysfunction in patients with benign childhood epilepsy as revealed by graph theory. PLOS ONE 10:e139228. doi: 10.1371/journal.pone.0139228
TFRs on scalp HD EEG would open new perspectives about the understanding of the mechanisms of IES generation and, through future studies, the causal role of local large-scale networks on changes to cognitive deficits.

\section{AUTHOR CONTRIBUTIONS}

Conceived and designed the experiments: EB, MM, PB, and FW. Performed the experiments: EB and MM. Contributed reagents/materials/analysis tool: $\mathrm{EB}, \mathrm{MM}$, and $\mathrm{FW}$. Wrote the paper: EB, MM, and FW. Read and accepted the manuscript: EB, $\mathrm{MM}$, and $\mathrm{PB}$, and $\mathrm{FW}$.

\section{ACKNOWLEDGMENTS}

The authors are grateful to the patients and their families for accepting to participate to our study. We thank also to the Amiens university hospital EEG technicians.

\section{SUPPLEMENTARY MATERIAL}

The Supplementary Material for this article can be found online at: http://journal.frontiersin.org/article/10.3389/fnins. 2017.00059/full\#supplementary-material

Figure S1 | Impact of varying slow wave amplitude on time-frequency analysis. (A) Simulated IES dipole in the right central sulcus, Locations, orientations. (B) Simulated IES with increasing slow wave amplitudes $(0,5,10,15$, $20 \mathrm{nAm})$. (C) The simulated scalp IES in average reference format. The solid lines indicate the signal + noise wave forms generated by the dipole sources indicated in (A). (D) Butterfly plot of the simulated spikes, Scalp potentials (33-channel montage) were simulated from dipole in order to correspond to field produced by the generator within right central sulcus. (Up-left) superimposing all channels. (E) The absolute power of time-frequency analysis is displayed. The $\mathrm{x}$-axis shows the time relative to the spike, the $y$-axis shows the frequencies. The intensities are displayed as a color-coded plot. (F) A time-frequency representation is shown where the power for each time is normalized to the mean power of the baseline epoch for that frequency, baseline period: [ $-1000 \mathrm{~ms} ;-600 \mathrm{~ms}]$. GTFR of the simulated IES demonstrated significant changes between 4 and $30 \mathrm{~Hz}$ in the right central sulcus simultaneously with the IES and an increase of power in low frequency band due to increase of the slow-wave amplitude of simulated spikes $(0,5,10,15,20 \mathrm{nAm})$.

Figure S2 | Transient change in Global Time-Frequency Representation (GTFR) in different scaling factors $(800,400$, and $200 \%)$.

Adebimpe, A., Aarabi, A., Bourel-Ponchel, E., Mahmoudzadeh, M., and Wallois, F. (2016). EEG resting state functional connectivity analysis in children with benign epilepsy with centrotemporal spikes. Front Neurosci. 10:143. doi: $10.3389 /$ fnins. 2016.00143

Alvarado-Rojas, C., Lehongre, K., Bagdasaryan, J., Bragin, A., Staba, R., Engel, J., et al. (2013). Single-unit activities during epileptic discharges in the human hippocampal formation. Front. Comput. Neurosci. 7:140. doi: 10.3389/fncom.2013.00140

Amiri, M., Lina, J. M., Pizzo, F., and Gotman, J. (2016). High frequency oscillations and spikes: separating real hfos from false oscillations. Clin. Neurophysiol. 127, 187-196. doi: 10.1016/j.clinph.2015.04.290

Auranen, T. (2002). Nonparametric Statistical Analysis of Time-Frequency Representations of Magnetoencephalographic Data. Master's thesis University of Technology, Department of Electrical and Communications Engineering Espoo.

Ayala, G. F. (1983). The paroxysmal depolarizing shift. Prog. Clin. Biol. Res. 124, 15-21. 
Beaumanoir, A., Ballis, T., Varfis, G., and Ansari, K. (1974). Benign epilepsy of childhood with Rolandic spikes. a clinical, electroencephalographic, and telencephalographic study. Epilepsia 15, 301-315.

Beaussart, M. (1972). Benign epilepsy of children with rolandic (centro-temporal) paroxysmal foci: a clinical entity. Study of 221 cases. Epilepsia 13, 795-811.

Bénar, C. G., Chauvière, L., Bartolomei, F., and Wendling, F. (2010). Pitfalls of high-pass filtering for detecting epileptic oscillations: a technical note on "false" ripples. Clin. Neurophysiol. 121, 301-310. doi: 10.1016/j.clinph.2009.10.019

Berg, A. T., Berkovic, S. F., Brodie, M. J., Buchhalter, J., Cross, J. H., van Emde Boas, W., et al. (2010). Revised terminology and concepts for organization of seizures and epilepsies: report of the ILAE commission on classification and terminology, 2005-2009. Epilepsia 51, 676-685. doi: 10.1111/j.1528-1167.2010.02522

Berg, P. (2006). Dipole Simulator. Version 3.3.0.6. Available online at: http://www. besa.de/updates/tools

Berg, P., and Scherg, M. (1994). A fast method for forward computation of multiple-shell spherical head models. Electroencephalogr. Clin. Neurophysiol. 90, 58-64.

Besenyei, M., Varga, E., Fekete, I., Puskás, S., Hollódy, K., Fogarasi, A., et al. (2012). EEG background activity is abnormal in the temporal and inferior parietal cortex in benign rolandic epilepsy of childhood: a LORETA study. Epilepsy Res. 98, 44-49. doi: 10.1016/j.eplepsyres.2011.08.013

Besseling, R. M., Overvliet, G. M., Jansen, J. F., van der Kruijs, S. J., Vles, J. S., Ebus, S. C., et al. (2013). Aberrant functional connectivity between motor and language networks in rolandic epilepsy. Epilepsy Res. 107, 253-262. doi: 10.1016/j.eplepsyres.2013.10.008

Cataldi, M., Avoli, M., and de Villers-Sidani, E. (2013). Resting state networks in temporal lobe epilepsy. Epilepsia 54, 2048-2059. doi: 10.1111/epi.12400

Davidson, A. C. (1999). Bootstrap Methods and their Application. Cambridge: Cambridge University Press.

De Curtis, M., and Avanzini, G. (2001). Interictal spikes in focal epileptogenesis. Prog. Neurobiol. 63, 541-567. doi: 10.1111/j.1535-7511.2006.00145.x

Elshoff, L., Groening, K., Grouiller, F., Wiegand, G., Wolff, S., Michel, C., et al. (2012).. The value of EEG-fMRI and EEG source analysis in the presurgical setup of children with refractory focal epilepsy. Epilepsia 53, 1597-1606. doi: 10.1111/j.1528-1167.2012.03587.x

Fahoum, F., Zelmann, R., Tyvaert, L., Dubeau, F., and Gotman, J. (2013). Epileptic discharges affect the default mode network-FMRI and intracerebral EEG evidence. PLoS ONE 8:e68038. doi: 10.1371/journal.pone.0068038

Fonseca, L. C., Tedrus, G. M., and Pacheco, E. M. (2007). Epileptiform EEG discharges in benign childhood epilepsy with centrotemporal spikes: reactivity and transitory cognitive impairment. Epilepsy Behav. 11, 65-70. doi: 10.1016/j.yebeh.2007.04.001

Garcia-Ramos, C., Jackson, D. C., Lin, J. J., Dabbs, K., Jones, J. E., Hsu, D. A., et al. (2015). Cognition and brain development in children with benign epilepsy with centrotemporal spikes. Epilepsia 56, 1615-1622. doi: 10.1111/epi.13125

Groening, K., Brodbeck, V., Moeller, F., Wolff, S., van Baalen, A., Michel, C. M., et al. (2009). Combination of EEG-fMRI and EEG source analysis improves interpretation of spike-associated activation networks in paediatric pharmacoresistant focal epilepsies. Neuroimage 46, 827-833. doi: 10.1016/j.neuroimage.2009.02.026

He, B. (1999). Brain electric source imaging: scalp Laplacian mapping and cortical imaging. Crit. Rev. Biomed. Eng. 27, 149-188.

Herrendorf, G., Steinhoff, B. J., Kolle, R., Baudewig, J., Waberski, T. D., Buchner, H., et al. (2000). Dipole-source analysis in a realistic head model in patients with focal epilepsy. Epilepsia 41, 71-80. doi: 10.1111/j.1528-1157.2000.tb 01508.x

Hoechstetter, K., Bornfleth, H., Weckesser, D., Ille, N., Berg, P., and Scherg, M. (2004). BESA source coherence: a new method to study cortical oscillatory coupling. Brain Topogr. 16, 233-238.

Huiskamp, G., van Der Meij, W., van Huffelen, A., and van Nieuwenhuizen, O. (2004). High resolution spatio-temporal EEG-MEG analysis of rolandic spikes. J. Clin. Neurophysiol. 21, 84-95.

Ibrahim, G. M., Cassel, D., Morgan, B. R., Smith, M. L., Otsubo, H., Ochi, A., et al. (2014). Resilience of developing brain networks to interictal epileptiform discharges is associated with cognitive outcome. Brain 137, 2690-2702. doi: 10.1093/brain/awu214
Ishitobi, M., Nakasato, N., Yamamoto, K., and Iinuma, K. (2005). Opercular to interhemispheric source distribution of benign rolandic spikes of childhood. Neuroimage 25, 417-423. doi: 10.1016/j.neuroimage.2004.11.040

Jacobs, J., Kobayashi, K., and Gotman, J. (2011). High-frequency changes during interictal spikes detected by time-frequency analysis. Clin. Neurophysiol. 122, 32-42. doi: 10.1016/j.clinph.2010.05.033

Jacobs, J., Levan, P., Chßtillon, C. E., Olivier, A., Dubeau, F., and Gotman, J. (2009a). High frequency oscillations in intracranial EEGs mark epileptogenicity rather than lesion type. Brain 132, 022-37. doi: 10.1093/brain/awn351

Jacobs, J., Levan, P., Moeller, F., Boor, R., Stephani, U., Gotman, J., et al. (2009b). Hemodynamic changes preceding the interictal EEG spike in patients with focal epilepsy investigated using simultaneous EEG-fMRI. Neuroimage 45, 1220-1231. doi: 10.1016/j.neuroimage.2009.01.014

Jacobs, J., Staba, R., Asano, E., Otsubo, H., Wu, J. Y., Zijlmans, M., et al. (2012). High-frequency oscillations (HFOs) in clinical epilepsy. Prog. Neurobiol. 98, 302-315. doi: 10.1016/j.pneurobio.2012.03.001

Jacobs, J., Vogt, C., LeVan, P., Zelmann, R., Gotman, J., and Kobayashi, K. (2016). The identification of distinct high-frequency oscillations during spikes delineates the seizure onset zone better than high-frequency spectral power changes. Clin. Neurophysiol. 127, 129-142. doi: 10.1016/j.clinph.2015.04.053

Jefferys, J. G., Menendez de la Prida, L., Wendling, F., Bragin, A., Avoli, M., Timofeev, I., et al. (2012). Mechanisms of physiological and epileptic HFO generation. Prog. Neurobiol. 98, 250-264. doi: 10.1016/j.pneurobio.2012.02.005

Kakisaka, Y., Iwasaki, M., Haginoya, K., Kanno, A., Tsuchiya, S., and Nakasato, N. (2011). Somatotopic distribution of peri-rolandic spikes may predict prognosis in pediatric-onset epilepsy with sensorimotor seizures. Clin. Neurophysiol. 122, 869-873. doi: 10.1016/j.clinph.2010.09.026

Kamada, K., Möller, M., Saguer, M., Kassubek, J., Kober, H., Wenzel, D., et al. (1997). Benign rolandic epilepsy investigated by magnetoencephalography. Biomed. Tech. 42, 185-187.

Kayser, J., and Tenke, C. E. (2006). Principal components analysis of Laplacian waveforms as a generic method for identifying ERP generator patterns: II. Adequacy of low-density estimates. Clin. Neurophysiol. 117, 369-380. doi: 10.1016/j.clinph.2005.08.033

Keller, C. J., Truccolo, W., Gale, J. T., Eskandar, E., Thesen, T., Carlson, C., et al. (2010). Heterogeneous neuronal firing patterns during interictal epileptiform discharges in the human cortex. Brain 133, 1668-1681. doi: 10.1093/brain/awq112

Kobayashi, K., Jacobs, J., and Gotman, J. (2009). Detection of changes of highfrequency activity by statistical time-frequency analysis in epileptic spikes. Clin. Neurophysiol. 120, 1070-1077. doi: 10.1016/j.clinph.2009.03.020

Kobayashi, K., Yoshinaga, H., Toda, Y., Inoue, T., Oka, M., and Ohtsuka, Y. (2011). High-frequency oscillations in idiopathic partial epilepsy of childhood. Epilepsia 52, 1812-1819. doi: 10.1111/j.1528-1167.2011.03169.x

König, P., Engel, A. K., and Singer, W. (1995). Relation between oscillatory activity and long-range synchronization in cat visual cortex. Proc. Natl. Acad. Sci. U.S.A. 92, 290-294.

Lantz, G., Grave de Peralta, R., Spinelli, L., Seeck, M., and Michel, C. M. (2003). Epileptic source localization with high density EEG: how many electrodes are needed? Clin. Neurophysiol. 114, 63-69. doi: 10.1016/S1388-2457(02)00337-1

Le Van Quyen, M., Soss, J., Navarro, V., Robertson, R., Chavez, M., Baulac, M., et al. (2005). Preictal state identification by synchronization changes in long-term intracranial EEG recordings. Clin. Neurophysiol. 116, 559-568. doi: 10.1016/j.clinph.2004.10.014

Lin, Y. Y., Shih, Y. H., Chang, K. P., Lee, W. T., Yu, H. Y., Hsieh, J. C., et al. (2003). MEG localization of rolandic spikes with respect to SI and SII cortices in benign rolandic epilepsy. Neuroimage 20, 2051-2061. doi: 10.1016/j.neuroimage.2003.08.019

Loiseau, P., and Beaussart, M. (1973). The seizures of benign childhood epilepsy with Rolandic paroxysmal discharges. Epilepsia 14, 381-389.

Makeig, S., Jung, T. P., Bell, A. J., Ghahremani, D., and Sejnowski, T. J. (1997). Blind separation of auditory event-related brain responses into independent components. Proc. Natl. Acad. Sci. U.S.A. 94, 10979-10984.

Manoochehri, M., Mahmoudzadeh, M., Osharina, V., and Wallois, F. (2017). Shedding light on interictal epileptic spikes: an in vivo study using fast optical signal and electrocorticography. Epilepsia. doi: 10.1111/epi.13689. [Epub ahead of print]. 
Neckelmann, D., Amzica, F., and Steriade, M. (2000). Changes in neuronal conductance during different components of cortically generated spikewave seizures. Neuroscience 96, 475-485. doi: 10.1016/S0306-4522(99) 00571-0

Nunez, P. L., and Srinivasan, R. (2006). A theoretical basis for standing and traveling brain waves measured with human EEG with implications for an integrated consciousness. Clin. Neurophysiol. 117, 2424-2435. doi: 10.1016/j.clinph.2006.06.754

Ochi, A., Otsubo, H., Shirasawa, A., Hunjan, A., Sharma, R., Bettings, M., et al. (2000). Systematic approach to dipole localization of interictal EEG spikes in children with extratemporal lobe epilepsies. Clin. neurophysiol. 111, 161-168. doi: 10.1016/S1388-2457(99)00208-4

Osharina, V., Ponchel, E., Aarabi, A., Grebe, R., and Wallois, F. (2010). Local haemodynamic changes preceding interictal spikes: a simultaneous electrocorticography (ECoG) and near-infrared spectroscopy (NIRS) analysis in rats. Neuroimage 50, 600-607. doi: 10.1016/j.neuroimage.2010.01.009

Panayiotopoulos, C. P., Michael, M., Sanders, S., Valeta, T., and Koutroumanidis, M. (2008). Benign childhood focal epilepsies: assessment of established and newly recognized syndromes. Brain 131, 2264-2286. doi: 10.1093/brain/awn162

Papp, N., and Ktonas, P. (1977). Critical evaluation of complex demodulation techniques for the quantification of bioelectrical activity. Biomed. Sci. Instrum. $13,135-145$.

Pataraia, E., Feucht, M., Lindinger, G., Aull-Watschinger, S., and Baumgartner, C. (2008). Combined electroencephalography and magnetoencephalography of interictal spikes in benign rolandic epilepsy of childhood. Clin. Neurophysiol. 119, 635-641. doi: 10.1016/j.clinph.2007.11.009

Rajagovindan, R., and Ding, M. (2008). Decomposing neural synchrony: toward an explanation for near-zero phase-lag in cortical oscillatory networks. PLoS ONE 3:e3649. doi: 10.1371/journal.pone.0003649

Ren, L., Kucewicz, M. T., Cimbalnik, J., Matsumoto, J. Y., Brinkmann, B. H., Hu, W., et al. (2015). Gamma oscillations precede interictal epileptiform spikes in the seizure onset zone. Neurology 84, 602-608. doi: 10.1212/WNL.0000000000001234

Simes, R. J. (1986). An improved Bonferroni procedure for multiple tests of significance. Biometrika 73, 751-754. doi: 10.1093/biomet/73.3.751

Srinivasan, R., Winter, W. R., Ding, J., and Nunez, P. L. (2007). EEG and MEG coherence: measures of functional connectivity at distinct spatial scales of neocortical dynamics. J. Neurosci. Methods 166, 41-52. doi: 10.1016/j.jneumeth.2007.06.026
Tang, Y. L., Ji, G. J., Yu, Y., Wang, J., Wang, Z. J., Zang, Y. F., et al. (2014). Altered regional homogeneity in rolandic epilepsy: a resting-state FMRI study. Biomed. Res. Int. 2014:960395. doi: 10.1155/2014/960395

Tong, S., and Thakor, N. V. (2009). Quantitative EEG Analysis Methods and Clinical Applications. Boston: Artech House.

Van Bogaert, P., Urbain, C., Galer, S., Ligot, N., Peigneux, P., and De Tiège, X. (2012). Impact of focal interictal epileptiform discharges on behaviour and cognition in children. Neurophysiol. Clin. 42, 53-58. doi: 10.1016/j.neucli.2011.11.004

van Klink, N. E., van't Klooster, M. A., Leijten, F. S., Jacobs, J., Braun, K. P., and Zijlmans, M. (2016). Ripples on rolandic spikes: a marker of epilepsy severity. Epilepsia 57, 1179-1189. doi: 10.1111/epi.13423

Vannest, J., Tenney, J. R., Gelineau-Morel, R., Maloney, T., and Glauser, T. A. (2015). Cognitive and behavioral outcomes in benign childhood epilepsy with centrotemporal spikes. Epilepsy Behav. 45, 85-91. doi: 10.1016/j.yebeh.2015.01.041

Verrotti, A., Filippini, M., Matricardi, S., Agostinelli, M. F., and Gobbi, G. (2014). Memory impairment and Benign Epilepsy with centrotemporal spike (BECTS): a growing suspicion. Brain Cogn. 84, 123-131. doi: 10.1016/j.bandc.2013.11.014

Vicente, R., Gollo, L. L., Mirasso, C. R., Fischer, I., and Pipa, G. (2008). Dynamical relaying can yield zero time lag neuronal synchrony despite long conduction delays. Proc. Natl. Acad. Sci. U.S.A. 105, 17157-17162. doi: $10.1073 /$ pnas.0809353105

Wirrell, E. C. (1998). Benign epilepsy of childhood with centrotemporal spikes. Epilepsia 39, S32-S41.

Xiao, F., An, D., Lei, D., Li, L., Chen, S., and Wu, X. (2016). Real-time effects of centrotemporal spikes on cognition in rolandic epilepsy: An EEG-fMRI study. Neurology 86, 544-551. doi: 10.1212/WNL.0000000000002358

Conflict of Interest Statement: The authors declare that the research was conducted in the absence of any commercial or financial relationships that could be construed as a potential conflict of interest.

Copyright (c) 2017 Bourel-Ponchel, Mahmoudzadeh, Berquin and Wallois. This is an open-access article distributed under the terms of the Creative Commons Attribution License (CC BY). The use, distribution or reproduction in other forums is permitted, provided the original author(s) or licensor are credited and that the original publication in this journal is cited, in accordance with accepted academic practice. No use, distribution or reproduction is permitted which does not comply with these terms. 